Aim To ensure appropriate community-based support for those recently bereaved, a large care provider in the West Midlands has established a team of Bereavement Visitors. The 20-strong team offer emotional support, provide information, education and coping strategies to bereaved relatives either on site/in the community. They also run individual and group therapy sessions to support those adjusting to the loss of a loved one.

Results Last year 241 people used the service, citing a range of benefits. In June 2018 the scheme was awarded the Queen's Award for Voluntary Service. This poster explores the drivers for the programme, discusses how volunteers are recruited, trained and supported and considers the impact and outcomes of the programme from a range of perspectives.

\section{P-11 RECRUITMENT OF BEREAVEMENT SERVICES USERS VIA HOSPICES: REFLECTIONS FROM THE BEREAVEMENT STUDY}

${ }^{1}$ Mirella Longo, ${ }^{1}$ Hannah Scott, ${ }^{1}$ Kathy Seddon, ${ }^{1} \mathrm{Jim}$ Fitzgibbon, ${ }^{1}$ Fiona Morgan, ${ }^{2}$ Sara Pickett, ${ }^{1}$ Anthony Byrne, ${ }^{1}$ Stephanie Sivell, ${ }^{1}$ Annmarie Nelson, ${ }^{1}$ Emily Harrop. ${ }^{1}$ Marie Curie Palliative Care Research Centre, Cardiff, UK; ${ }^{2}$ Swansea Centre for Health Economics, Swansea, UK

\subsection{6/bmispcare-2018-hospiceabs.36}

Background The quality of the recruitment process has a fundamental influence on the potential value of research outputs. The bereavement study aimed to develop a set of core outcomes specific to bereavement support services for bereaved adults. A consensus methodology involving all the key stakeholders (including service users) was used to address the research questions. Nine hospices in the UK were approached to facilitate recruitment of study participants. We report on the recruitment of service users.

Methods Service users were asked to answer to a two-round DELPHI questionnaire (Hasson, Keeney \& McKenna, 2000). Past service users could not be contacted because hospices would not normally retain the details of the people who have left their services. We relied on hospice staff who regularly saw bereavement support service users to identify users who were on their way to recovery and were able to reflect on the outcomes that were important when assessing the value of the service they received. Hospices were given two months to carry out recruitment.

Results Hospice-based health professionals helped us refine inclusion and exclusion criteria, time from loss was not an inclusion/exclusion criteria. Some of the hospices had lower recruitment rates mainly due to the tight time schedule set for recruitment. One in three of the service users replied to the first questionnaires and, of these, 90\% participated to the second round of questionnaires.

Conclusions Hospice staff played an important role in supporting the recruitment of bereavement service users, but researchers need to allow enough time for efficient recruitment. Earlier engagement allowed active influence over the recruitment process. Hospital 'internal' readiness to recruit is an important factor. Both the literature (Bentley \& O'Connor, 2015; Akard, Gilmer, Miller et al., 2014) and practice suggest that bereavement study participants are themselves the best judges on when to participate in research studies and time from loss is not an important inclusion/exclusion criteria.

\section{P-12 PUBLIC INVOLVEMENT (PI) IN A STUDY OF BEREAVEMENT SUPPORT SERVICES FOR ADULTS}

${ }^{1}$ Kathy Seddon, ${ }^{1} \mathrm{Jim}$ Fitzgibbon, ${ }^{1}$ Barbara Harrington, ${ }^{2}$ Fiona Morgan, ${ }^{1}$ Hannah Scott, ${ }^{1}$ Stephanie Sivell, ${ }^{3}$ Sara Pickett, ${ }^{1}$ Mirella Longo, ${ }^{1}$ Anthony Byrne, ${ }^{1}$ Annmarie Nelson, ${ }^{1}$ Emily Harrop. ${ }^{1}$ Marie Curie Research Centre, Cardiff, UK; ${ }^{2}$ Specialist Unit for Review Evidence, Cardiff, UK; ${ }^{3}$ AWTTC, Cardiff, UK

\subsection{6/bmjspcare-2018-hospiceabs.37}

Introduction Bereaved adults may develop complicated grief reactions associated with mental and physical health problems and good support services are vital. This research included a systematic review and the development of a core outcome set for bereavement support service research in palliative care. The collaborative relationship (PI) with the Research Partners (RPs) was fully planned and then documented through reflective logs. PI was central to this research.

Aim To detail the RPs' contribution to this study.

Methodology RPs helped refine the research question. Following this a study protocol was agreed that set out the steps needed to ensure that: (1) Research design and methods used were appropriate for bereaved adults; (2) All documentation and outputs were accessible to 'lay' readers; (3) RPs 'experience based' expertise was embedded in ongoing planning, outputs and ongoing research design; (4) The experience for RPs was worthwhile leading to new skills and knowledge detailed in the reflective logs. Reflective log sheets were used to capture how well this was achieved. Researchers and RPs completed these independently. Their reflections were shared at quarterly meetings and used to inform each stage of the research.

Results The reflective logs confirmed that steps 1 to 5 were carried out. RP inputs achieved the planned impact (e.g. the questionnaires held hardly any missing data, reflecting content and face-validity). Enhancement of the bereavement research through planned, documented RP collaboration was thus confirmed.

Conclusion Well-planned and documented PI can enhance the quality of health care research. Researchers felt that the solid collaboration with the research partner broadened their perspective and signalled a clear departure from a simple tick-box exercise. Research Partners confirmed the rich learning gained through participation.

\section{P-13 A PILOT STUDY: EXPLORING FAMILY BEREAVEMENT EXPERIENCES WHEN A MEMBER OF THE FAMILY HAS DEMENTIA}

${ }^{1}$ Nikki Archer, ${ }^{2}$ Alison Pooler. ${ }^{1}$ St Giles Hospice, Lichfield, UK; ${ }^{2}$ Keele University, Keele, UK

10.1136/bmjspcare-2018-hospiceabs.38

Bereavement support for families is an integrated and essential component of palliative care (Hudson, Hall, Boughey et al., 2017), although a growing evidence base for the provision of services exists (National Bereavement Alliance, 2017), the need to broaden our understanding to reflect broader family and sociological perspectives has been identified (Stroebe \& Schut, 2015). Despite the growing incidence of dementia (Alzheimer's Society, 2017), little is known about the bereavement experience of people with dementia (PWD), (Watanabe \& Suwa, 2017). 